\title{
LA-UR-20-27212
}

Approved for public release; distribution is unlimited.

Title: Initial Experiences with HPE Apollo80 NSP-1 (A64FX)

Author(s): $\quad$ Pritchard, Howard Porter Jr.

Intended for: $\quad$ presentation for Riken/SNL collaborators

Issued: 2020-09-15 
Disclaimer:

Los Alamos National Laboratory, an affirmative action/equal opportunity employer, is operated by Triad National Security, LLC for the National Nuclear Security Administration of U.S. Department of Energy under contract 89233218CNA000001. By approving this article, the publisher recognizes that the U.S. Government retains nonexclusive, royalty-free license to publish or reproduce the published form of this contribution, or to allow others to do so, for U.S. Government purposes. Los Alamos National Laboratory requests that the publisher identify this article as work performed under the auspices of the U.S. Department of Energy. Los Alamos National Laboratory strongly supports academic freedom and a researcher's right to publish; as an institution, however, the Laboratory does not endorse the viewpoint of a publication or guarantee its technical correctness. 


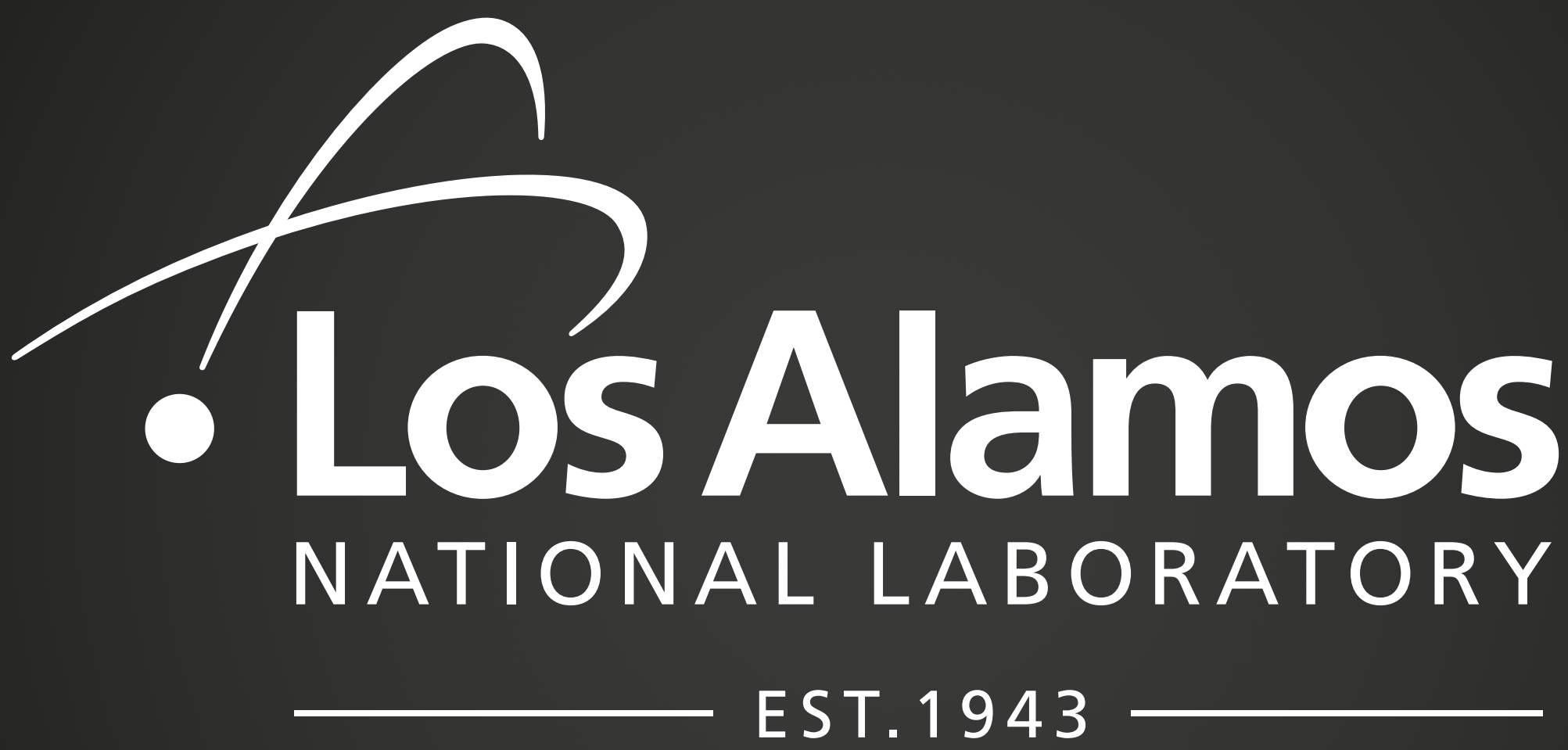




\section{Initial Experiences with HPE Apollo80 NSP-1 (A64FX)}

Howard Pritchard howardp@lanl.gov 


\section{HPL/Stream baselining}

○ Got reasonable results

- No slow nodes

- No thermal issues (we had a lot of these with Cavium TX2 in early days) 


\section{ASC apps status}

- EAP, LAP, SAP, Partisn codes and deps built using GCC 10.2.0, passing at least some unit tests

- Mixed success so far with HPE CCE sve 10.0.0 compiler. Where it can be used tending to be best.

- LAP and EAP can't be built with Alinea flang - not an aarch64 issue

o Performance issues 


\section{Mini-apps}

- Laghos, SNAP, pennant, clamr, xsbench, cloverleaf, tealeaf, branson. Have most of these building with GNU and CCE. Mixed performance results.

- Clamr - hit a compiler bug with GCC 10.2.0 


\section{Performance problems}

o Consistently seeing high backend stall counts irrespective of whether application is cache friendly or not. 


\section{SNAP Cray craypat summary}

\section{NSP1: A64FX}

Total

\begin{tabular}{lc} 
Thread Time & $3,009.189541$ secs \\
L1D_CACHE_REFILL & $27,889,231,282$ \\
L1D_CACHE & $2,769,095,692,372$ \\
INST_RETIRED & $7,440,467,616,712$ \\
CPU_CYCLES & $5,349,564,724,029$ \\
L2D_CACHE & $60,303,751,021$ \\
L2D_CACHE_REFILL & $14,611,691,746$ \\
STALL_BACKEND & $2,684,139,720,866$ \\
Backend stall cycles / Cycles & $50.2 \%$ \\
\hline Retired Inst per Clock & 1.39 \\
\hline MIPS 2,472.72M/sec & \\
D1 cache hit,miss ratios $99.0 \%$ hits $\quad 1.0 \%$ mis: \\
D2 cache hit,miss ratio $75.8 \%$ hits $24.2 \%$ mis \\
$=====================================$
\end{tabular}

Grind Time (nanoseconds) $\quad 3.5208 E+01$

SNAP is faster on A64FX, esp. using CCE, but not that much faster, even though vectorizes well. Note grind time on skylakegold is 10 nanoseconds.

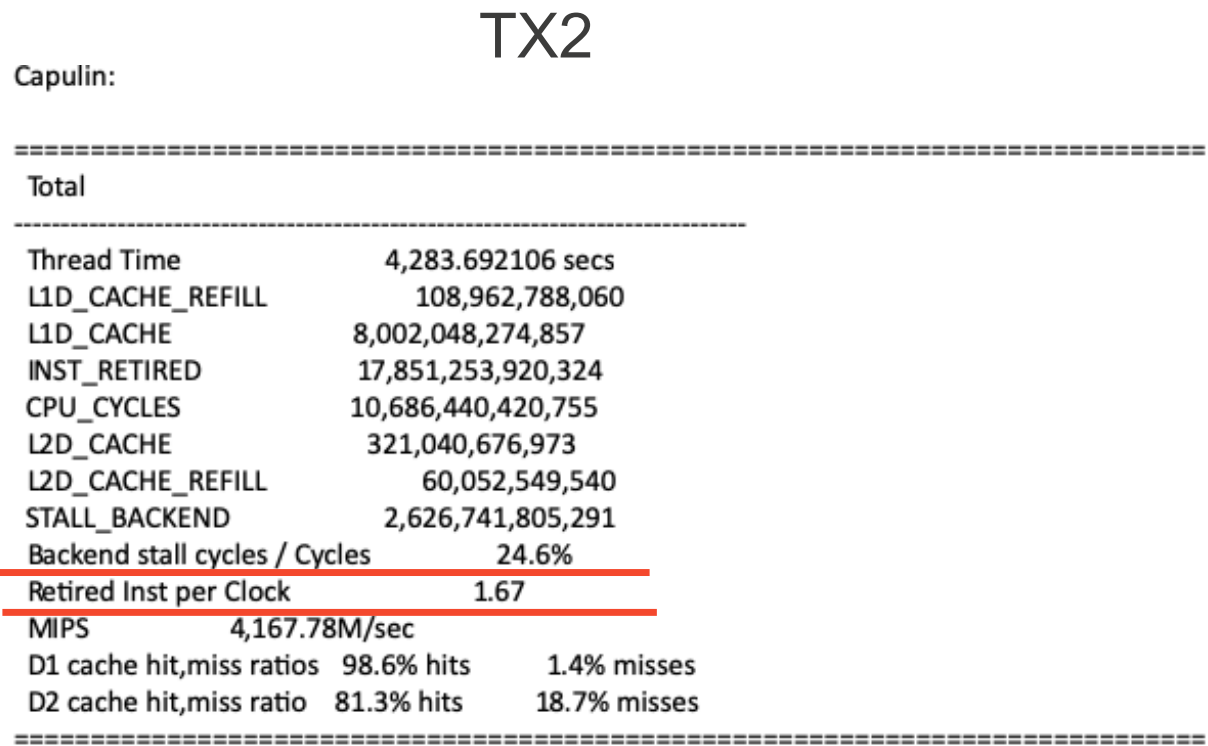

Grind Time (nanoseconds) $\quad 5.0122 \mathrm{E}+01$ 


\section{Cloverleaf Cray craypat summary}

\section{A64FX}

\section{NSP-1}

Thread Time

L1D_CACHE_REFILL

L1D_CACHE

INST_RETIRED

CPU_CYCLES

L2D_CACHE

L2D_CACHE_REFILL

STALL_BACKEND

Backend stall cycles / Cycles

Retired Inst per Clock

MIPS

$296.89 \mathrm{M} / \mathrm{sec}$

D1 cache hit,miss ratios $86.8 \%$ hits

D2 cache hit,miss ratio

$67.6 \%$ hits
662.801682 secs

$9,969,686,617$

$75,318,344,492$

196,756,388,166 <--------

$1,171,844,874,604$

$20,634,455,555$

$6,681,590,112$

$1,100,149,051,305$

\section{TX2}

TX2

Thread Time

L1D_CACHE_REFILL

L1D_CACHE

INST_RETIRED

CPU_CYCLES

L2D_CACHE

L2D_CACHE_REFILL

STALL_BACKEND

Backend stall cycles / Cycles

Retired Inst per Clock

MIPS

$2,331.98 \mathrm{M} / \mathrm{sec}$

D1 cache hit,miss ratios $84.4 \%$ hits

D2 cache hit,miss ratio $86.6 \%$ hits

$13.4 \%$ misses

।

case $4: 3840 \times 3840$ cells with $87-$ steps

CCE: -hcache 0 flex mp=rigorous

CCE: -hcacheo flex mp=rigorous

CCE: -hcache日

GNU: -03

GNU: -03 -funroll-loops

GNU: -0fast -funroll-loops

ARM: -03 -fno-unsafe-math-optimizations

ARM: -0fast -fno-unsafe-math-optimizations

$\begin{array}{rlll}424 & \mathrm{sec} & \text { PASSED } & \text { ThunderX2 } \\ 647 \mathrm{sec} & \text { PASSED } & \text { NSP-1 } \\ 564 \mathrm{sec} & \text { PASSED } & \text { NSP-1 } \\ 1072 \mathrm{sec} & \text { PASSED } & \text { NSP-1 } \\ 1065 \mathrm{sec} & \text { PASSED } & \text { NSP-1 } \\ 837 \mathrm{sec} & \text { PASSED } & \text { NSP-1 } \\ 773 \mathrm{sec} & \text { PASSED } & \text { NSP-1 } \\ 769 \mathrm{sec} & \text { PASSED } & \text { NSP-1 }\end{array}$

417.581831 secs

$37,095,984,820$

$237,720,730,800$

973,439,003,899<----- clos

$1,040,438,646,857$

$36,901,343,768$

$4,948,962,669$

$377,495,562,093$
$36.3 \%<---m$ much

$0.94<--5.5 x$

\section{$15.6 \%$ misses}




\section{Next Steps}

- Get CCE non-sve compilers installed on system

○ needed for some ASC app dependencies

- Get Fujitsu compiler installed on system.

- Try GCC 11 head-of-master

- Use caliper or more advanced craypat experiments to better understand high backend stall counts

- Has been suggested that there's some problem with A64FX store forwarding performance

o Try mini-apps on Riken FX700 and Fugaku 


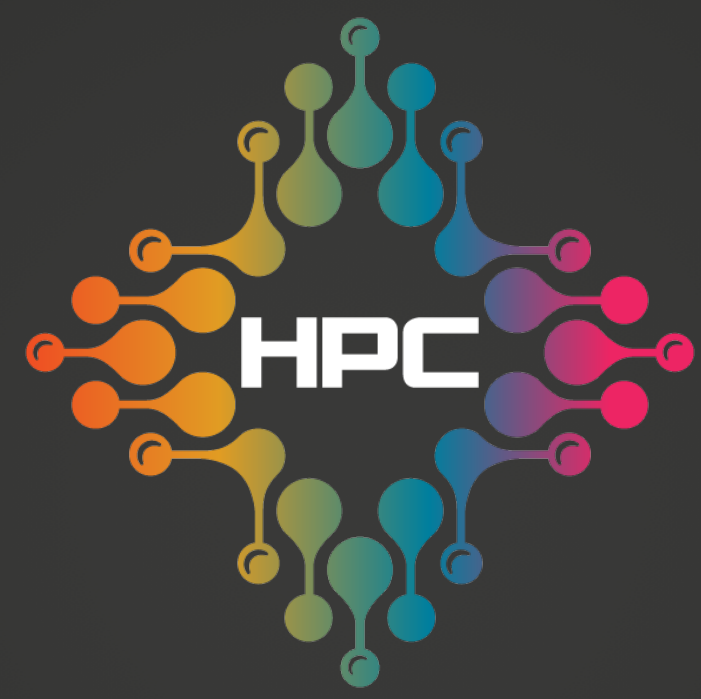

Over 70 years at the forefront of supercomputing 